\title{
Bindung, Mentalisierung, Psychiatrie und Psychotherapie
}

\author{
Herwig Oberlerchner
}

Eingegangen: 24. September 2016 / Angenommen: 19. September 2017 / Online publiziert: 13. Oktober 2017

(c) Der/die Autor(en) 2017. Dieser Artikel ist eine Open-Access-Publikation.

Zusammenfassung Der Autor, Psychiater und Psychoanalytiker, fasst im vorliegenden Beitrag die Ergebnisse der Bindungstheorie (Bindungsmuster, Bindungstypen, Fremde Situation) zusammen und stellt das Mentalisierungskonzept vor. Fußend auf diesen Erkenntnissen ergeben sich Konsequenzen für den psychiatrischen und psychotherapeutischen Alltag, die in wenigen Punkten umrissen werden.

Schlüsselwörter Bindung · Mentalisierung · Psychiatrie $\cdot$ Psychotheapie

\section{Attachement, mentalization, psychiatry and psychotherapy}

Summary The author, psychiatrist and psychoanalyst, summarizes in the following article the principles of the attachement theory (attachement patterns, types of attachement, strange situation) and presents the concept of mentalization. Based on this findings consequences for the psychiatric and psychotherapeutic work are outlined in a few points.

Keywords Attachement · Mentalization · Psychiatry · Psychotherapy

\section{Bindungstheorie - Ein Überblick}

Der englische Kinderarzt, Kinderpsychiater und Psychoanalytiker John Bowlby hat sich in seinem um-

\author{
H. Oberlerchner $(\bowtie)$ \\ Abteilung für Psychiatrie und Psychotherapie, Klinikum \\ Klagenfurt am Wörthersee, Feschnigstr. 11, 9020 Klagenfurt, \\ Österreich \\ herwig.oberlerchner@kabeg.at \\ H. Oberlerchner \\ Seegasse 56, 9020 Klagenfurt, Österreich
}

fangreichen Werk (1975 [1969], 1976 [1973], 1983 [1980]) mit Überlegungen zur Bindung auseinandergesetzt. Unter Bindung wird die Neigung eines Individuums verstanden, unter bedrohlichen, beängstigenden, fremden Bedingungen Nähe zu suchen und Kontakt herzustellen (durch Weinen, Anklammern, Lächeln, Hilfe suchen, Schreien, Nachfolgen ...). Es geht also um ein Gefühl der Sicherheit spendenden Nähe zu einem Gegenüber, um ein Bedürfnis nach Nähe. Es handelt sich dabei - so Bowlby - um ein eigenständiges, angeborenes Motivationssystem (neben Sexualität und Aggression), das durch die Ergebnisse der Säuglings- und Kleinkindforschung, Verhaltensforschung und der Psychoanalyse bestätigt wird.

Ein Kleinkind bindet sich als Ausdruck seines Wunsches nach Sicherheit an eine Person und durch die Reaktionen dieser Bindungsfigur auf seine Signale entsteht ein „inner working model“ (Bowlby 1975 [1969]), ein Modell, auf das das Kind in bedrohlichen Lebenssituationen zurückgreift.

Mary Ainsworth, eine amerikanische Entwicklungspsychologin, untersuchte in den 70er Jahren diese Bindungsmuster bei 12-18 Monate alten Kindern im Rahmen einer standardisierten Versuchsanordnung, der "fremden Situation" (Ainsworth et al. 1978). Diese besteht aus acht standardisierten Episoden zu je drei Minuten, kreisend um die zentrale Szene des Abschiedes und der Wiederkehr der Mutter. Das Verhalten des Kindes in dieser komplexen Situation wird gefilmt und anschließend analysiert. Primär wurden je nach beobachtetem Verhalten beim Kind nach Weggehen und Rückkehr der Bezugsperson drei charakteristische Verhaltensweisen unterschieden: sichere Bindung, unsicher-vermeidende Bindung, unsicher-ambivalente Bindung, später kam eine vierte Kategorie dazu. Diese Kategorie wurde zunächst als cc-Kategorie („cannotclassify“) bezeichnet, später als unsicher-desorganisiertes Bindungsmuster. Untersucht man größere Kol- 
lektive in der Allgemeinbevölkerung, so erhält man folgende prozentuelle Verteilung von Bindungsmustern: 55-23-8-15\% (van Ijzendoorn 1992).

Erwachsene können bezüglich ihrer Bindungsrepräsentanz im Rahmen des Adult Attachement Interviews - entwickelt 1985 von der amerikanischen Entwicklungspsychologin Mary Main und ihren Mitarbeiterinnen (Main et al. 1985) - befragt werden. Dieses semistrukturierte Interview umfasst 18 Fragen zu Kindheit, Bezugspersonen, emotionaler Atmosphäre, Trost bei Kummer, Einfühlung in elterliches Verhalten, Verlusterlebnissen, Beziehung $\mathrm{zu}$ den eigenen Kindern und dauert ungefähr eine Stunde. Vier Bindungstypen und ihre Charakteristika werden in Bindungsinterview unterschieden: autonom (free-autonomous), verstrickt (entangled-enmeshed), distanziert (dismissing), und der von unverarbeiteten Objektverlusten beeinflusste Bindungstyp (unresolved). Eine zeitlich wesentliche kürzere Untersuchungsmöglichkeit stellt das Adult-Attachement-Projective-PictureSystem dar (George und West 2012), das es bereits in einer deutschen Version gibt.

Viele erwachsene PatientInnen mit psychischen Problemen wurden nun schon in Bezug auf ihre Bindungserfahrungen mit dem AAI untersucht, die Mutter-Kind-Interaktion zwischen psychisch kranken Müttern und deren Kindern beforscht, und sowohl bei den psychisch kranken Erwachsenen als auch bei deren untersuchten Kindern finden sich markante Verschiebungen der prozentuellen Verteilung in Richtung unsichere bzw. desorganisierte Bindung, eine Möglichkeit, die Häufung psychischer Erkrankungen in Familien zu erklären, aber auch die transgenerationale Weitergabe psychischer Strukturen.

Bindungsmuster sind in hohem Ausmaß abhängig von der Feinfühligkeit (schnelles, angemessenes Reagieren auf kindliche Bedürfnisse) der primären Bezugsperson. Deren Verhaltensweisen (im Optimalfall positiv, sensitiv und vorhersagbar) bestimmen die Bindungsqualität der ersten Monate. Bindungsmuster geben also Auskunft über die Interaktionsgeschichte und haben später als Bindungsrepräsentanzen beim Erwachsenen einen starken Einfluss auf das Sozialverhalten, die Ärztin/Arzt-TherapeutIn-PatientInBeziehung, auf Aufmerksamkeit, Phantasie und Kreativität, Aggression, Misstrauen, Nähe-Distanz, IchStärke, Frustrationstoleranz, Krankheitshäufungen und die Fähigkeit in neuen und herausfordernden Lebenssituationen Sicherheit und Selbstvertrauen zu bewahren.

Je unsicherer die Bindungsrepräsentanz, desto höher ist auch das spätere Risiko, psychopathologisch relevante Probleme zu entwickeln bei Auftreten zusätzlicher Belastungsfaktoren.

Umgekehrt sind nun feinfühlige, klar-strukturierte, verlässliche und haltgebende Bindungsangebote Chancen für PatientInnen in psychiatrischen Institutionen. Das bisher Gesagte zu den bindungstheoretische Modellen hat nun einige Implikationen für die psychiatrische Behandlung und die Begegnung zwischen den PatientInnen und den MitarbeiterInnen der verschiedenen Berufsgruppen der in psychiatrischen Einrichtungen tätigen multiprofessionellen Teams.

\section{Implikation für die stationäre psychiatrische Betreuung}

1. Wir können von einer hohen Zahl von unsicher gebundenen Menschen unter den stationären PatientInnen ausgehen, daher ist auf ein Betreuungskontinuum achten. Die PatientInnen profitieren auch im stationären oder sonstigen institutionellen Betreuungsansätzen vom Kontinuum im Kontakt mit dem therapeutischen Personal.

2. Auch oder gerade bei allfällig notwendigen Wiederaufnahmen ist auf TherapeutInnenkonstanz zu achten.

3. Bezugspflege, klare Zuständigkeiten, ein transparenter Wochenplan und ein klar strukturierter Tagesablauf kommen bindungsirritierten Menschen entgegen.

4. Regionalisierung vor Spezialisierung. Bindungstheoretisch ist der Regionalisierung (Betreuung von PatientInnen aus bestimmten Regionen an der immer selben Station) vor der Spezialisierung (Schwerpunktstationen) der Vorzug zu geben.

5. Die psychiatrische Betreuung von PatientInnen sollte primär Beziehungsmedizin sein. Bei aller Wichtigkeit von Leitlinien, Algorithmen, Standards und Erfolgsmessung ist die Stärke der Psychiatrie echte und kongruente, zwischenmenschliche und möglichst glückende Interaktion und Kommunikation, um korrigierende Bindungserfahrungen zu ermöglichen. Dafür braucht es ausreichende menschliche Ressourcen.

6. „Gute Qualität in der Medizin ist nicht mit finanziellen Anreizen zu erreichen, sondern mit Zeit und Ruhe für eine gute Betreuung"(Maio 2015, S. 1356).

7. Es ist daher wichtig in Arbeitszufriedenheit, Teamgeist und Achtsamkeit zu investieren. Einzel- und Teamsupervision müssen neben vielen anderen Möglichkeiten zur Verbesserung von Selbstreflexion und Introspektion Standard sein.

8. Beziehungsmedizin mit den Chancen für Korrektur missglückter Bindungserfahrung und für glückende Mentalisierungsprozesse wird unter atmosphärisch und architektonisch ansprechenden Rahmenbedingungen besonders gut gelingen.

\section{Mentalisierung - Ein Überblick}

Unter Mentalisierung versteht man - im weitesten Sinn - die Fähigkeit des Menschen über sich selbst und andere zu reflektieren. Man kann durch Mentalisierung eigenes Handeln und das Handeln anderer in einem bedeutungsvollen Zusammenhang mit mentalen Zuständen und Vorgängen sehen. Hinter dem Handeln können Intentionen und Motive erkannt und 
benannt werden wie Gefühle, Gedanken, Bedürfnisse, Wünsche, Sehnsüchte, Begründungen, Bedeutungen fußend auf der individuellen Lebenserfahrung und Biographie und über diese mentalen Zustände kann wiederum nachgedacht werden (Fähigkeit zur Metakognition) (Fonagy 1998). Es gibt Überlappungen zu den allgemeinen Begriffen wie Introspektions- und Reflexionsfähigkeit, Feinfühligkeit und Empathiefähigkeit.

Mentalisierung bedeutet eine Vorstellung $\mathrm{zu}$ besitzen, welche aktuellen oder auch zeitlich zurückliegenden Hintergründe und Ursachen für das eigene oder das Verhalten anderer vorliegen könnten. Persönliches Verhalten, das Verhalten des anderen und der interpersonale Anteil können so interpretiert werden. Mentalisieren gibt daher Orientierung und hilft bei der Kontrolle in der Interaktion mit anderen Menschen (vor allem in affektiv schwierigen Situationen), unterstützt in der eigenen Emotions- und Selbstregulation und ermöglicht größere emotionale und soziale Kompetenz (Stichwort: emotionale Intelligenz). Es ermöglicht Innehalten, Aufschieben und Nachdenken in affektiv aufgeladenen Situationen.

Die Fähigkeit zur Mentalisierung und Affektregulation entwickelt sich beim Kind ab den ersten Lebensmonaten und am besten auf dem Boden einer stabilen und tragfähigen Beziehung zu den primären Bezugspersonen, einer sicheren Bindung. Mary Main (1991) hat festgestellt, dass die metakognitiven Fähigkeiten sicher gebundener Kinder größer sind als die unsicher gebundener Kinder. Eltern, die selbst als Kinder sicher gebunden waren, haben in einem höheren Prozentsatz sicher gebundene Kinder (Dornes 1998). Eltern mit einer sicheren Bindungsrepräsentanz sind eher in der Lage diese reflexive Haltung einzunehmen, was Peter Fonagy auch durch die Entwicklung eines eigenen Instrumentes (der self-reflecting-scale, einer Ergänzung zum adult-attachement-interview) untersuchen und belegen konnte (Fonagy 1998). Die Mentalisierungsfähigkeit der Mutter - die Fähigkeit dem noch nicht intentionalen Kind Deutungen absichtsvollen Handelns verbal oder non-verbal anzubieten hat einen bestimmenden Einfluss auf das Bindungsverhalten des Kindes. Diese Form der mentalisierenden Interaktion mit dem Kind basierend auf einer sicheren Bindung hat wiederum einen großen Einfluss auf neurophysiologische Regulationssysteme wie Stresshormone, die wiederum Hirnreifung und Entwicklung regulieren.

Nimmt man biologische und psychosoziale Faktoren zusammen, findet sich also ein Teufelskreis: Gestörte Bindung beeinträchtigt Hirnreifung und Mentalisierung. Die daraus resultierende erhöhte Vulnerabilität führt zu persistierenden Störungen in Beziehungen, die ihrerseits das Mentalisierungsdefizit und die arretierte Hirnreifung perpetuieren (Potthoff 2008).
In einem neuen Ansatz haben „Peter Fonagy und seine Arbeitsgruppe (Fonagy 1991) entwicklungspsychologische, psychoanalytische, traumabezogene und neurobiologische Erkenntnisse auf den Prüfstand der empirischen Säuglings-, Kleinkind- und Psychotherapieforschung gestellt und mit der Theory of Mind (ToM) zum Mentalisierungsansatz amalgamiert" schreibt Bohm (2010). Tatsächlich finden sich im Mentalisierungskonzept viele bekannte Ansätze:

- Objektbeziehungstheoretische Überlegungen der Kleinianischen Schule (Stichwort schizoide Position, depressive Position).

- Symbolisierung (Freud, Segal, Bion, Winnicott).

- Das Konzept der projektiven Identifikation (Klein, Kernberg, König, Ogden).

- Das Containment-Konzept von Bion (die feinfühlige Mutter ist in der Lage besonders die negativen Affekte des Kindes aufzunehmen und wandelt diese Beta-Elemente in für das Kind erträglichere AlphaElemente um).

- Das Desomatisierungskonzept von Max Schur.

- Entwicklungspsychologische Erkenntnisse wie Interaffektivität und Affektspiegelung (Gergely, Watson).

- Bindungstheorie (siehe oben) ...

Dieses Konzept hat sich weiter entwickelt und ist nun einerseits ein entwicklungspsychologisches, in dem innovativ die Pole der affektiv-emotionalen und kognitiven Dimension des Erlebens des Kindes angenähert werden, aber auch ein Erklärungsansatz für die Psychopathologie bestimmter PatientInnen mit spezifischen Störungsbildern und darüber hinaus auch ein Ansatz für die Psychotherapie (Stichwort: Mentalisierungsbasierte Therapie - MBT) (z. B. Fonagy et al. 2004 und Bateman und Fonagy 2008 [2004]).

\section{Förderung von Mentalisierung}

Für den Erwerb der Fähigkeit zur Mentalisierung gibt es glückende und störende Rahmenbedingungen. Zusammenfassungen finden sich in den bereits erwähnten Arbeiten von Fonagy und Bateman oder auch bei Dornes (2002, 2004).

Das Kleinkind kann Emotionen (primäre Affekte wie Ärger, Ekel, Furcht, Freude oder Traurigkeit ...) eventuell auch in Kombination mit physiologischen Veränderungen des Vegetativums ausdrücken im Sinne mimisch-gestischer, physiologischer und motorischer Schablonen, weiß aber nichts über die emotionalen Hintergründe. In der glückenden Eltern-KindInteraktion werden die Eltern diesen Gefühlszustand erkennen und spiegeln.

Diese Beantwortung ist meist überzeichnet, etwas künstlich und übertrieben (Stichwort Babysprache), auch um die Aufmerksamkeit beim Kind zu erhöhen. Die Eltern akzentuieren die Äußerungen des Säuglings, was als Markierung bezeichnet wird. Der Säugling merkt, dass der gespiegelte Affekt nicht echt, son- 
dern gespielt und entfremdet ist (referenzielle Entkoppelung) und bezieht das im Gesicht des Erwachsenen wie in einem Monitor abgebildete (deswegen der Bezug zum Biofeedback) auf die eigene Affektlage (referenzielle Verankerung). Im Lauf der weiteren Entwicklung wird diese Interaktion immer lebendiger und detaillreicher.

Das Kind erlebt so nach und nach die Unterscheidungsfähigkeit zwischen Innen- und Außenwelt, die Fähigkeit zur Selbstregulation und Beruhigung, die Erkenntnis von Getrenntheit und die Fähigkeit zur Symbolisierung. Die sekundäre symbolische Selbstregulierung geschieht über die Generierung von Interaktionsrepräsentanzen. Das heißt, dass neben dem eigenen, primären Gefühl immer auch das durch die Spiegelung der Eltern induzierte und modifizierte Gefühl in Sinne einer sekundären Repräsentanz abgerufen wird. Damit wird das Kind vom realen Gegenüber und der externen Darstellung unabhängig, kann nun auch im Spiel oder in der Phantasie innere Zustände darstellen und modifizieren. Nicht mehr das Gesicht der Eltern ist die externe Darstellung sondern die (fantasierte) Spielfigur, die Kommentare der Eltern oder ihr (markiertes) Mitspielen werden einbezogen, das Geschehen so kontrollierbar und spielerisch modifiziert.

Es entstehen im Rahmen der Affektspiegelung eine Subjektrepräsentanz (ein Bild vom eigenen Erleben in bestimmten Situationen), eine Objektrepräsentanz (ein Bild vom eventuell nur symbolisch repräsentierten Gegenüber in bestimmten Situationen) und eine Interaktionsrepräsentanz (ein Bild vom jeweiligen interagierenden Verhalten in diesen Situationen) und eine zunehmende Kompetenz bzgl. der Regulationsfähigkeit von Affekten. Selbstzustände werden mit Bilder und Gedanken verbunden, im Spiel und in der Fantasie durchgearbeitet.

\section{Störungen der Mentalisierung}

Durch mangelhafte Spiegelung, unsichere oder desorganisierte Bindung bei traumatisierenden Lebensumständen, Übergriffen, unbewältigten elterlichen Erfahrungen u. ä. leidet die Fähigkeit zur Mentalisierung. Es kommt zum Reaktivieren pathologischer Interaktionsrepräsentanzen, mit Beeinträchtigung von Exploration und Integration gerade in Stresssituationen. Dadurch kann es auch zu affektiver Überflutung, Impulsdurchbrüchen, Kontrollverlusten, Intrusionen, dissoziativen Phänomenen oder gar Psychosen usw. kommen mit permanenter Bedrohung des Kohärenzgefühles. Diese Phänomene finden wir auch häufig bei Menschen mit Borderline-Persönlichkeitsstörung aber auch bei höher strukturierten Menschen, die in evtl. psychosozial getriggerten affektiven Ausnahmezuständen auf Stufen der noch zu erwähnenden Mentalisierungsentwicklung regredieren.

Die Mentalisierung kann konkret gestört werden durch abnorme Spiegelprozesse, die es verhindern, dass das Kind sich als abgegrenztes, kohärentes Wesen empfindet. Diese können natürlich auch durch genetisch-konstitutionelle Faktoren seitens des Kindes verursacht sein, zum Beispiel in der Maximalvariante tiefgreifender Entwicklungsstörungen (Autismus) oder durch das Unvermögen der Eltern. Unmarkiertes Spiegeln verhindert, dass das Kind zwischen eigenem Erleben und dem der Bezugspersonen unterscheiden lernt. Es erfolgt keine Affektregulierung oder spielerischer Umgang mit dem Affekt, eher eine Eskalation und keine Vermittlung von Symbolisierung. Vermeidung oder starke Beschränkung der Affektspiegelung kann zur Schizoidie oder stark körperbezogener Selbstwahrnehmung (Stichwort Psychosomatik) führen, inkongruentes Spiegeln wiederum zu Narzissmus und dem Falschen Selbst nach Winnicott (Potthoff 2008).

Bis zur Entwicklung einer reifen Mentalisierung im fünften Lebensjahr finden sich einige Stadien der Mentalisierungsentwicklung. Diese Modi stehen dem Kind parallel zur Verfügung, werden erst gegen Ende des 5. Lebensjahres integriert. Im teleologischen Stadium (bis zum Alter von 1,5 Jahren) ist nur das konkrete Ergebnis von Handlungen bedeutsam, Gedanken und Gefühle stehen im Hintergrund, die innere Wirklichkeit ist noch blass. Eigene Regungen und die Regungen anderer werden noch nicht als durch mentale Zustände verursacht erkannt. Dem Verhalten anderer wird - beim Erwachsenen - keine ihn betreffende Bedeutung zugemessen. Dornes (2004) spricht von Bedeutungsblockierung oder unkorrekter Mentalisierung. Nur konkrete und greifbare Handlungen beruhigen und mildern innere Spannungszustände.

Drei kurze Fallvignetten sollen solche Vorstadien der Mentalisierung darstellen:

Vignette I: Eine Patientin erlebt immer wieder heftige Selbstwertgefühlseinbrüche verbunden mit Anspannung und tiefer Unzufriedenheit mit ihrem Äußeren. Die zehn Nasenkorrekturen und vielen Lippenunterspritzungen entlasten sie nur kurzfristig. Nach dem letzten Eingriff wird der nächste geplant. Im Stadium der Äquivalenz (,,psychic equivalence modus") werden Innen und Außen gleichgesetzt, Gedanken und Gefühle werden wahr. Gedanken haben ja beim Kleinkind denselben Effekt wie ein wirkliches Geschehen. Immer wieder gibt sich die Patientin der Hoffnung hin, dass durch eine konkrete Veränderung ihr Äußeres betreffend, sich Zufriedenheit und Entspannung im Inneren einstellen.

Vignette II: Eine sexuell schwer traumatisierte Patientin erlebt eine permanente Bedrohung durch heftige Intrusionen im Sinne von flash-backs. Aus Angst vor äußerer (und innerer) Bedrohung verlässt sie kaum das Zimmer, nimmt nur sehr wenig Nahrung zu sich, kann nur bei lauter, die Umgebungsgeräusche übertönender Musik schlafen. Im Stadium des As-ob-Modus („pretend modus“) sind Phantasie und Realität wie im intensiven Spiel des Kindes nicht völlig getrennt. Erwachsene Betroffene leben in ei- 
ner Scheinwelt, es kann zu einer affektiv-kognitiven Dissonanz kommen.

Vignette III: Ein junger Mann wird mit den ganzen Körper erfassenden Schüttel- und Zuckanfällen eingeliefert. Er kann nicht gehen und stehen. Diese konversionsneurotische Symptomatik, die er völlig affektentkoppelt zur Schau stellt, drückt sein unbewusstes innerseelisches Dilemma im Sinne eines chronifizierten Autonomie-Bindungskonfliktes aus. Die Fähigkeit zur Mentalisierung und Metakognition im Sinne von Introspektion und Selbstreflexion sind nicht vorhanden.

Typisch für diese Menschen mit Borderline-Persönlichkeitsorganisation ist, dass sie in konfliktgeladenen Situationen zu Hyperarousal (mit Aktivierung der Stressachse), einer Re- und Hyperaktivierung alter Bindungsrepräsentanzen und zu Mentalisierungsversagen mit Regression auf oben genannte Vorstufen neigen, ein Kreislauf der sich auch in therapeutischen Beziehungen wiederholen kann. Ziel ist die Entwicklung hin zu einem reflektierenden Modus, der Außen und Innen, Affekte, Symbolisierung, Phantasien und Metakognition vereint.

Dornes fasst die bei Borderlinepatienten beobachtbaren Mentalisierungsprobleme nun folgendermaßen zusammen:

1. Borderlinepatienten sind teilweise im AlsOb-Modus des Denkens fixiert, teilweise im Äquivalenzmodus, teilweise alternieren sie zwischen beiden; 2. Borderlinepatienten hemmen die Mentalisierung, indem sie auf den teleologischen Modus regredieren. 3. Sie alternieren zwischen Bedeutungsblockierung (im teleologischen Modus) und unkorrekter und/oder überaktiver Mentalisierung“ (Übertreibung alternativer Schauplätze um abzulenken; Anm. des Autors) ... „Der gemeinsame Nenner all dieser Varianten ist, dass Borderlinepatienten sich schwer tun, eigene und fremde mentale Zustände (korrekt) zu identifizieren (Dornes 2004).

Implikation für die Gruppentherapie (auch im Rahmen stationär psychiatrischer Behandlung)

Aufgrund der Erkenntnisse des Mentalisierungskonzeptes sollten Modifikationen in der Einzel- und Gruppentherapie vorgenommen werden, diese bedeuten aber keinen radikalen Perspektiven- bzw. Paradigmenwechsel (Potthoff und Moini-Afchari 2012), sondern wichtige Ergänzungen und Modifikationen der Behandlungstechnik. Für das einzeltherapeutische Setting mit Borderline-PatientInnen liegt bereits ein Manual von Bateman und Fonagy aus dem Jahr 2004 vor, die Wirksamkeit dieses Ansatzes ist auch bereits mehrfach belegt.

Nach all dem Gesagten wird klar, dass BorderlinepatientInnen aber auch höher strukturierte KlientInnen in Krisen mit Regression auf frühe Mentalisie- rungsmodi oder in affektiven Ausnahmezuständen von einer Haltung der/s Therapeutin/en nicht profitieren, die geprägt ist von Passivität, Zurückhaltung und Abstinenz. Auch wird für viele PatientInnen die Arbeit mit Widerstand, Übertragung und Deutung zu früh und eventuell eine Überforderung darstellen. Das heißt die Haltung und Präsenz des Therapeuten wird eine andere sein müssen. Dies auch deshalb, weil die Gruppen zwar durch die große Anzahl multipler Spiegelungsangebote die Mentalisierung fördern und durch das Interesse am anderen anfänglich im Konkreten und Bewussten bleiben wird, gleichzeitig aber das Fremde und durch die Affizierbarkeit und interaktionelle Potenz von Menschen mit BorderlinePersönlichkeitsorganisation Ängste und Regression auslösen kann.

In mehreren Dimensionen ist daher eine Modifikation der therapeutischen Grundhaltung notwendig (Potthoff und Moini-Afchari 2012; Bolm 2010, 2008). Dazu ein Überblick.

1. Die therapeutische Grundhaltung sollte geprägt sein von Interesse, Neugier, forschendem Nachfragen und Zurückhaltung bzgl. Deutung. Dem Prinzip (Übertragungs-) Deutung steht das Prinzip Frage gegenüber.

2. Aktives Anregen der Selbstreflexionsfähigkeit durch Klärung und Nachfragen.

3. Spiegelnde, markierende und validierende (anerkennende) Interventionen fußend auf dem Entwicklungskonzept der Mentalisierung.

4. Unterbrechen von Mentalisierungsbrüchen um das Regredieren in alte Modi zu vermeiden.

5. Arbeit im und Fokus auf das Hier und Jetzt. Arbeit mit bewussten und vorbewussten Inhalten.

6. Verzicht auf zu intensive Darstellung von Traumatisierungen. Vermeiden zu hoher Affektintensität.

7. Aktives Gestalten eines Klimas von Sicherheit, Interesse, Anregung, Holding und Containing. Nur bei sicherer Bindung gelingt Mentalisierung.

8. Sorgfältige Aufklärung. Erzeugen von Bezogenheit und Gruppenkohärenz, Struktur und Transparenz.

9. Verwenden einer einfachen und gut verständlichen Sprache nahe an der Alltagssprache.

MBT ist ein manualisiertes Behandlungskonzept (Bateman und Fonagy 2008 [2004]), für eine Klientel, die sich in der Krankheitsschwere in der Regel von der einer psychotherapeutischen Praxis unterscheidet und im deutschsprachigen Raum eher im stationären, teilstationären oder psychiatrischen Institutsambulanzkontext vorkommt. MBT-Bestandteile sind dennoch gut anwendbar in jeder Praxis, können jedoch bei relativ stabilen Patienten mit herkömmlichen psychodynamischen oder verhaltenstherapeutischen Techniken kombiniert werden. Damit handelt es sich nicht um MBT, wohl aber um Psychotherapie, in denen die Mentalisierungsfähigkeit gefördert wird (Bolm 2008). 
Das heißt, dass MBT im engeren Sinn zwar für PatientInnen mit Traumafolgeerkrankungen und Borderline-Persönlichkeitsstörungen entwickelt und an dieser PatientInnengruppe auch untersucht wurde, aber einige Prinzipien und Grundhaltungen auch in anderen Gruppen und auch bei stationär behandelten Menschen verwendet werden können.

Potthoff und Moini-Afchari schließen ihre Arbeit mit dem Satz: „Die Alternative kann also nicht heißen MBT oder Gruppenanalyse, sondern MBT in der Gruppenanalyse“ (2012).

\section{Fazit}

Ich möchte abschließend noch einen Schritt weitergehen. Bisher flossen bindungstheoretische und Mentalisierungskonzepte eher nur in die Anwendung und Modifikation (gruppen-) psychotherapeutischer Ansätze ein. Geht man aber davon aus, dass ein großer Anteil stationär behandelter Patienten unsicher gebunden sind und Mentalisierungsdefizite haben, müssen wir diesen Menschen im psychiatrischen Behandlungsalltag Rahmenbedingungen atmosphärischer und kommunikativer Natur anbieten, die ihnen Bindungssicherheit vermittelt und damit ihre Fähigkeit zur Metakognition fördert. Ein Transfer der Erkenntnisse der Bindungstheorie und der Mentalisierung in die psychiatrischen Anstalten scheint mir daher ein Gebot der Stunde.

Interessenkonflikt H. Oberlerchner gibt an, dass kein Interessenkonflikt besteht.

Open Access Dieser Artikel wird unter der Creative Commons Namensnennung 4.0 International Lizenz (http:// creativecommons.org/licenses/by/4.0/deed.de) veröffentlicht, welche die Nutzung, Vervielfältigung, Bearbeitung, Verbreitung und Wiedergabe in jeglichem Medium und Format erlaubt, sofern Sie den/die ursprünglichen Autor(en) und die Quelle ordnungsgemäß nennen, einen Link zur Creative Commons Lizenz beifügen und angeben, ob Änderungen vorgenommen wurden.

\section{Literatur}

Ainsworth, M., etal. (1978). Patterns of attachement. A psychological study of the strange situation. Hillsdale: Erlbaum.

Bateman, A., \& Fonagy, P. (2008). Psychotherapie der Borderline Persönlichkeitssörung. Gießen: Psycho-Sozialverlag.

Bolm, T. (2008). Mentalization-Based Treatment (MBT) in der stationären und ambulanten Gruppenpsychotherapie. In M. Hirsch (Hrsg.), Die Gruppe als Container. Mentalisierung und Symbolisierung in der analytischen
Gruppentherapie(S. 144-167). Göttingen:Vandenhoeck\& Ruprecht.

Bolm, T. (2010). Mentalisieren und Mentalisierungsbasierte Therapie(MBT). Psychotherapie, 15, 58-62.

Bowlby, J. (1975). Bindung. München: Kindler. Erstausgabe 1969

Bowlby, J. (1976). Trennung. München: Kindler. Erstausgabe 1973

Bowlby, J. (1983). Verlust, Trauer und Depression. München: Kindler. Erstausgabe 1980

Dornes, M. (1998). Bindungstheorie und Psychoanalyse. Konvergenzen und Divergenzen. Psyche, 52(4), 299-348.

Dornes, M. (2002). Die emotionale Welt des Kindes (3. Aufl.). Frankfurt: Fischer.

Dornes, M. (2004). Über Mentalisierung, Affektregulierung und dieEntwicklungdesSelbst. Forum der Psychoanalyse, 20(2), 175-199.

Fonagy, P. (1991). Thinking about thinking: some clinical and theoretical considerations in the treatment of a borderline patient. International Journal of Psychoanalysis, 72, 639-656.

Fonagy, P. (1998). Metakognition und Bindungsfähigkeit des Kindes. Psyche, 52, 349-368.

Fonagy, P., Jurist, E., Gergely, G., \& Target, M. (2004). Affektregulierung, Mentalisierung und die Entwicklung des Selbst. Stuttgart: Klett-Cotta.

George, C., \&West, M. (2012). The adult attachment projective picture system. New York: Guilford.

van Ijzendoorn, M. (1992). Intergenerational transmission of parenting.: a review of studies in nonclinical populations. Developmental Review, 12,76-99.

Main, M. (1991). Metacognitive knowledge, metacognitive monitoring and singular (coherent) versus multiple (incoherent) models of attachement:findings and directionsfor future research. In C. M. Parks, J.Stevenson-Hinde\&J.Harris (Hrsg.), Attachement across the life cycle (S. 127-159). London, New York: Routledge.

Main, M., Kaplan, N., \& Cassidy, J. (1985). Security in infancy, childhood, and adulthood: a move to the level of representation. In I. Bretherton \& E. Waters (Hrsg.), Growing points of attachment theory and research. Monographs of the Society for Research in Child Development, Vol. 50, No. 1/2. (S.66-104).

Maio, G. (2015). „Ich tue das richtige nur gegen Belohnung“. Eine Kritik an Pay-for-Performance in der Psychiatrie. Nervenarzt, 86, 1349-1357.

Potthoff, P. (2008). Mentalisierung und gruppenanalytische Behandlungstechnik. In M. Hirsch (Hrsg.), Die Gruppe als Container. Mentalisierung und Symbolisierung in der analytischen Gruppentherapie (S. 86-116). Göttingen: Vandenhoeck\&Ruprecht.

Potthoff, P., \& Moini-Afchari, U. (2012). Mentalization based Treatment (MBT) in der Gruppe - neues Paradigma oder alter Wein in neuen Schläuchen. Gruppenpsychother. Gruppendynamik, 48, 198-214. 